\title{
LYRIK AUF DEM LANDE - EIN BEITRAG ZUR DEUTSCH-BRASILIANISCHEN LITERATUR \\ Reinaldo Bosmmann
}

In Stäten mit einem sturkeren Kulturbetrieb und der Auswah 1 von mehreren Bildungswegen trifft man bfter auf Menschen, die ihren Gedanken, GefUhlen, empfangenen Eindrucken, Umweltein flussen, Hoch- und Tiefstimmungen schbpferischen Ausdruck geben. In den Ballungszentren von Industrie, Handel, pulsierendem Verkehr, Touristik, Theater-und Vortragsleben empfangen sie sicher reichere Impulse, Anregungen, haben mehr Beobachtungs und Anschaungsobjekte als Menschen in der Einsamkeit- soweit am in unserer zeit von einer solchen noch sprechen kann-, in lundicher Stille. Wenn der Gegensatz von Stadt zu Land auch in Brasilien durch den technologischen Fortschrite, durch den Ausbau von Verkehrsnetzen langsam zu schwinden beginnt, so bleiben doch die Bewohner lundlicher Gebiete den Stadrmenschen gegenuber, was die Offerte an Kultur, Ausbildungsmbglichkeiten, Zerstreuung und Unterhaltung anbelangt, noch stark im Nachieil. Hier wie dort wird zur Lebensbehauptung harte Arbeit heute verlangt, wenn man nicht gerade an irdischen Gutern geseg net ist, und der schbpferischen kraft sind keine Grenzen gesetzt. Bei Besuchen, Vortragsteisen, Diskussions- und Leseaben den auf dem Lande begegnet man kenschen, die sich von anderen abheben: Neben schwerer kbrperlicher Tagesarbeit, meist Bauern arbeit, pflegen sie das schungeistige in oft kyrglich bemesse 
nen und sich abgerungenen ifussestunden, schreiben prosa oder Lyrik, fungieren als Festredner bei den verschiedensten Gelegenneiten, die dem 1 undichen Leben auch zahlreich gegeben sind; sie sind oft Mittelpunkt des kulturellen Geschehens und wer den dainer auch manchmal Dichter, nicht im abwertenden Sinn, genant. Solche Nebenfunktionen dieser Menschen und ihre Ausstrahlung auf die Kolonie in kultureller Hinsicht kbnnen nicht hoch genug bewertet werden. Es sind meistens Menschen, denen in der Jugend ein anderer Beruf vorschwebte, die dann aber auf der Ackerscholle geblieben sind, sei es aus Tradition oder uuch infolge eines ungunstigen Schicksals, das dann den Aufbruch in eine andere Richtung bestimmte.

In der Nuhe von Castro lebt der 73 juhrige ERICH KARL QUAST, der aus einem Pfarrhaus in der Neumark ${ }^{1}$ stamt und seit 1921 in Paranä als Bauer und Huhnerzllchter ansyssig ist. Gegen Ende des 1. Weltkrieges, mit dem Notabitur versehen, musste er noch den feldgrauen Rock anziehen. Dem Wunsch seines Vaters, auch pearrer zu werden, hat er, wie er jetzt in Versen bekennt, damals nicht entsprochen:

Wenn selber $Z$ weifel mich verzehren, wie mocht' ich andere belehren?

Was mir erstrebenswert geblieben?

Den Nuchsten wie sich selber lieben!

(1) Teil der Mark Brandenburg, Bstlich der Oder gelegen. 
Lange vor den Donauschwaben in Guarapuava hat Bauer Quast in Paraná Weizen angebaut. Wenn Hollünder zu Besuch kamen und die grossen, prachtigen Weizenfelder sahen, bemerkten sie: "As in Holland." So war es auch richtig, als es um den Weizenanbau der Donauschwaben in den ersten Jahren nicht gut stand, dass sich die Schweizer Hilfe den Fachmann Quast zu Vortrugen auf diesem Gebiete in die Kolonien um Guarapuaa holte. Auch beim Aufbau der hollandischen Kolonie Castrolanda hat Quast aus Idealismus mitgewirkt und sich um das Hollynderwerk einige Ver dienste erworben.

Sein Hobby ist das Schachspiel. Er hat den Ruf, ein ausgezeich neter schachspieler zu sein. Fruher spielte er oft seine Partien gegen mehrere Gegner zugleich. Doch an einem richtigen Tournier hat er leider noch nie teilgenommen.

Als Ausgleich zur schweren kbrperlichen Arbeit schreibt er, hüufig in nuchtlichen stunden, Gedichte ${ }^{2}$, Theaterstucke 3 Anekdoten, Sinnspruche und Erzylungen, die er bescheiden schriftiche Bemlhungen nennt. Quast schreibt meitens nach dem Motto:

\section{Beschau' das Leben mit Humor, dann komt dir vieles anders vor:}

(2) Er verbffentlichte einen Gedichtband wit dem Titel"Der Hase im Unterrock", Curitiba, $1969,77 \mathrm{~s}$, gedruckt bei Imprimax Ldta. Darin befindet sich Humoristisches, Besinnliches Balladeskes und Sinnprichliches.

(3) "Der neue Hut", eine Bauernkombie, und "Drei Zwerge wollen heiraten", ein Murchenspiel fur Kinder. Beide stllcke wurden mehrmals in Terra Nova aufgefuhrt, sind aber noch nicht veruffenticht worden. 
Aus seiner Feder fliessen humorvolle Verse, die auf den ersten B $1 \mathrm{ick}$ wegen ihrer Einfachheit anspruchslos erscheinen, aber im Kern doch viel Lebensweisheit, Erfahrung und einen versteckten Sinn enthalten, der sich als liebevolle Satire auf Mitmen schen, Begebenheiten im Dorfleben, auf negative Umwelterscheinungen entpuppt. Quast, naturverbunden, ist ein guter Beobachter von Menschen und ihrem Tun, von dbrflichen Vorkommissen, die sofort schnell die Runde machen. Diese Bereiche geben ihm sehr oft die Motive zu seinen lustigen Reimereien, andere entnimmt er aus Zeitungsmeldungen, IUsst sich von stoffen aus BUchern, von Erzuhlungen und Berichten aus dem Freundeskreis inspirieren.

Auf die Gedichte, die Quast in der Broschure "Der Hase im Unter rock" - sie beruien vielfach auf mit Dichtung und Wahrheit ver mischten Begebenheiten -, herausgab, soll hier nicht weiter eingegangen werden; sie sind lesenswert und daher Freudenspender. Wichtiger scheint es, hier die Lyrik ${ }^{4}$ zu bieten, die noch nicht publiziert wurde oder nur einem kleinen kreis bekannt ist.

\section{Der Falter und die Rosenblute}

Dort fliegt ein Falter vor mir her, setzt dreist sich auf die Rosenblute, fragt gar nicht, ob erlaubt es war, fuhrt sich den Nektar zu Gemlite.

(4) Die meisten seiner Gedichte hat Quast ohnehin im original verschenkt, ohne vorher Abschriften angefertigt zu haben. 
Dann taumelt er berauscht hinweg, hab' ihn ob diesen nicht gescholten. Weil ihm der Diebstahl Lebenszweck, bleib' ihm solch Tun auch unvergolten.

\section{Mäte 1 chen-Lied}

Ich wollt', ich whr' dein Muntelchen, so schmiegt' ich mich wohl voller lust, dass ich den Herzchlag spluren kbnnt', an deine hold geformte Brust.

Ich wollt', ich wyr' dein Muntelchen, blieb es flur immer, lebenslang. Dass ich dich hullen, whrmen durft', whr mir der allerschbnste Dank

\section{Wenn zwei dasselbe tun}

Es sitzt ein Starmatz auf dem Ast, er pfeiftund trillert, wie's ihm passt. $O b$ er auch stets dasselbe singt, wie es doch hychst erbaulich klingt.

Den Menschen doch, der intoniert und nur dasselbe moduliert, den kritisiert man: Armer Tropf: Bei dem fehlt es bestimmt im Kopf. Man Uberdenkt es und begreift: von Wichtigkeit ist, wer was pfeift. 


\section{Friedensbemthungen}

Den Frieden mit friedlichen Mitteln zu finden, die Gytter selbst wussten's und wissen's nicht, wie?

Uns Sterblicien Iiessen sie's, dies zu ergrunden und doch, auch der Weise erkundet es nie.

$$
\underline{\text { Vom Baum gefallen }}^{5}
$$

In des Baumes hychsten Zweigen, wo die sch sieht amn einen Leichtsinn steigen, jeder ahnt $z u$ welchen zwecken.

Dem, der Lockendes betrachtet, schwindet leicht das Uberlegen, dies indem er nicht beachtet, sich mit vorsicht zu bewegen.
Also ging's auch dem Genannten, einmal trat sein Fuss ins Leere. Erdwhrts nach dem wohlbekannten Grundsatz fiel des kurpers Schwere.

(5) Vor 11 Jahren Uberlebte Quast diesen Sturz. Dass sein Erdenweg damals nicht juh abgebrochen wurde, ist weniger der Hrztichen Kunst als mehr seiner Willenskraft und dem unverwllstichen Humor zur Lebensbehauptung zuzuschreiben. 
Linksseitig total zerschlagen, was an Knochen innenwendig, ward zum Doktor er getragen;

tot scinen er menr als lebendig.

Dazu der GerUchte LUgen:

Jener, der vom Baum geglitten, liege in den letzten Zugen, habe demnucinst ausgelitten.

Mittwochs drang dies Totgelate zu der ilachbarin Kathrine:

"Sterbe er doch nur nicit heute", seufze sie mit trloer iliene.

"will er absolut von hinnen, will's ihn von der Erde treiben, solite, dieses $z$ u beginnen, mbglichst fur den Sanstag bleiben.

Sonntags wird er dann begraben. Nur der Tag gibt Zeit und Ruhe, hinter seinem Sarg zu traben, schwarz das Kleid und sciwarz die Sçune."

Doch entgegen allen Trumen und trotz sicherem Erwarten turnt er wieder auf den Bumen, 
auf den höchsten in dem Garten.

Ist jedoch Kathrinens wegen

samstags nur im Baum zu sehen,

weil ein Sonntag mehr gelegen,

hinter seinem Sarg zu gehen.

Sein Theaterstlick "Der neue Hut" hat als Vorwort folgenden Achtsilber:

Ein Bauer kauft sich einen Hut.

Wie es so geht, er passt nicht gut.

Uber die weitere kandlung dieses stllckes informieren die Reimpare:

Des Bauern Frau ist mitverwickelt,

das StUck wird somit schon verzwickelt.

Ein Ladenschwenge 1 , ein gerieb'ner,

geiojrt dazu, ein ganz durchtrieb'ner.

Nun tritt in des Geschehens Lauf

ein Husserst nettes Yudchen auf.

Der Hbrer merkt es, er verspurt,

jetzt wird es noch menr kompliziert.

Hinzu kommt noch des Mladchens Vater,

ein Geck erscheint auch im Theater.

Und all dies Drum und all dies Dran,

was dieser spricht, was der getan,

es flhrt zum Zveck, zu dem es soll,

der iut sitzt letzthin wundervoll. 
Sein 2. Stllck "Drei Zwerge wollen heiraten", ein 'lurchen, ganz in Versen geschrieben, wurde von kindern aufgefunrt, darunter auch ein kleiner Akteur, der das Deutsche nur gebrochen sprach; er spielte seine Rolle ebenso gut wie die anderen deutscinsprachigen Mini-schauspieler:

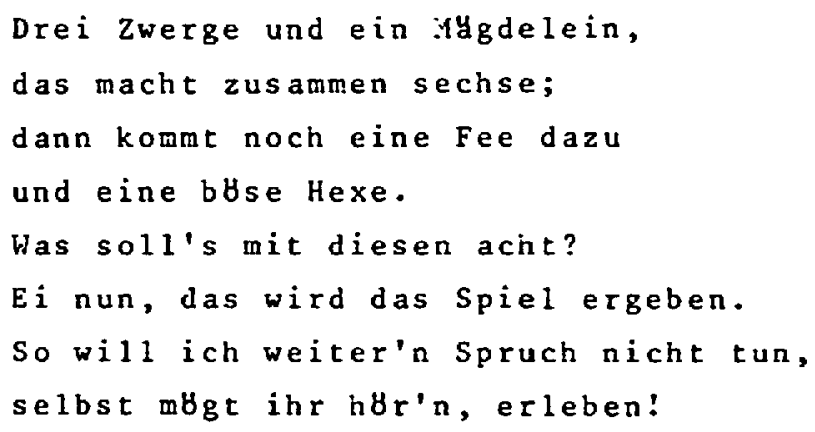

Dass Gott nicht Uber alle Geschbpfe herrscht, ist im vierzei1er "Entdecktes Spruchlein aus dem Schreibtisch" zu lesen:

Eindeutig sei es festgestellt:

Der liebe Gote herrscht in der welt.

In einem nur stimmt's nicht genau:

Beim Mann regiert gar oft die Frau.

Um die Ehre des weiblichen Geschlechts zu retten, schreibt Quast Uber seine angetraute Frau, die hier stellvertretend fur ihre Artgenossinnen steht:

Sie heisst Johanna :largareth, wie es im Pass urkundich steht. 
Was fur sie einnimmt, fur sie spricht:

Sie kocht recht gut - und dichtet nicht.

Quast, der die deutsche Dichtung in Brasilien ${ }^{6}$ um sourwlich sig gesunde und humorvolle Beitrage bereichert hat, setzt sich fur sein Schaffen durch eigene Aussage ein bleibendes Denkmal :

Der Hand, die sonst die kacke schwingt,

fehlt's kunstvoll hinzuschreiben, und wenn sie doch die Feder zwingt, ist's oft ein hart Betreiben.

In Terra Nova lebt ANTON Scliulte. Er ist Bauer und betreibt vorwiegend Milciwirtscilaft. In ibln an 9 . Liovenier 1915 geboren, besuchte er zunachst die Volksschule, spater das Gymasium bis zum Einhjhrigen in seiner Geburtsstadt. Die ungunstigen finanziellen Verhaltinisse des Elternhauses ${ }^{7}$ und die

(6) Es fullt auf, dass in den uns zur Verfugung gesteliten Gedichten kein einziges luber ein Thema aus Brasilien und Deutschland vorhanden ist, wie es oft der Fall bei Carl Fried, Karl Niederhut und Clemente Raul Einsiedel ist. Haben ihn Geburtsland und Wahlheimat nicht inspiriert?

(7) 4 Geschwister studierten. 
Wirtschaftskrise im Jahre $1932^{8}$ erlaubten ihm nicht, das Gymnasium mit dem Reifezeugnis absuschliessen und ein hochschul studium zu beginnen. Er enstschied sich fur die Landwirts chaft, 9 arbeicete als Eleve in vorbildlichen landwirtschaftlichen Betrieben im Rheinland, bis eine Erjschaft inm im Jahre 1935 die Auswanderung nach Brasilien ermbglichte. Auch ohne das Geschenk der Erbschaft hatte er schon vorher geplant, Deutschland wegen der braunen Diktatur zu verlassen. In Begleitung seiner Mutter, eines glteren Bruders und seiner beiden Schwestern kam er nach Terra Nova,um auf "Neuer Erde"ein neues Leben zu beginnen. Hiergrlindete er Hof und Familie, wurde ses haft und war den zeiten wechselnden Glucks und der Ruckschlage ausgesetzt. Am Gedeihen dieser kleinen Gemeinde nimt er regen Anteil, seiner stundigen Mitarbeit sind Kirche, Schule und Kulturverein gewiss. Uber Einladungen, bei gegebenen Anlussen Festreden zu halten, ist er jedesmal erfreut.Gern ubernimmt er auch Rollen fur die dortige Laiennspielgruppe und das krip penspiel, das alljuhrlich Uber die Buhne im Festsaal von Terra Nova geht.

Seit seiner Schulzeit pflegt er die Neigung zur Dichtkunt. Dass er sich ihr nicht mehr widmen kann, liegt an den Anforderungen, die die Landwirtschaft an ihn stellt. Schulte ist eine

(8) Herrschende Arbeitslosigkeit, besonders in akademischen Be rufen.

(9) Von einem alten westfylischen Erbhof stamme sein Vater. 
durch und durch religibse Natur, die sich in seinen Versen offenbart.Den Mitmenschen Freude $z u$ spenden, ist fur ihn ede1ste Pflicht eines aufrechten Christen. Sein Stammland, besonders kBln und den Rhein, kann er nicht vergessen. Doch auch seine Wahlheimat, seine engere Umgebung, die Dorfgemeinschaft bieten sich ihm als Themen an, das Schbne, Gute und waine zu besingen. Schulte ist ein konservativer Mensch. Die alten und guten Werte wie Glaube, Treue, Verantwortung, Erfurcht, Beken nertum und opfermut haben fur ihn noch Glltigkeit.

Anton Sciulte hat nicht viel geschrieben, fast gar nichts ver Bffenticht. Die meisten seiner Gedichte bat er verschenkt, Abschriften von ihnen existieren nicht. Was vorliegt, sind in erster Linie viele Festreden, lie er an Gedenktagen, besonders zum 25. Juli (Dia do Colono) gehalten hat. Sie weisen eine sehr gute Prosa auf. Was an Lyrik zur Verfugung steht, sind me stens Gedichte von betrucinticher Lunge. Sie kunnen hier nicht vollstandig, sondern nur auszugsweise, wiedergegeben wer den :

\section{Morgendummerung}

Zart graut der Tag Uber schlafender Erde. Wie schbn ist die Geburt des Licht's, als ob sich wiederhole Gottes Vort: Es werde! Und purpurn whist der Sonnenball wie aus dem Nichts.

Gebet und Arbeit kluger Sinn das sind die Zauberstube dieses Lebens. 
Bedien' dich ihrer, dein ist der Gewinn!

Und dein Bestreben ist wohl nie vergebens.

Mein Sohn, es ist nicht alles Gold, was glanzt.

Willst Du Vertrauen schenken, schau nur wer!

So manche Hydchenstirne ist zwar reich bekrynzt,

es fehlt jedoch der Reinheit hehres Diaden. II

Dort oben syuselt und raunet der hind, dort, wo Herz und Sinne sich weiten.

Unglaubliche Form in stein geprugt

als Zeugen vergangener zeiten. 12

Was mir aus Jugendtagen tief in der Seele klingt,

was hier im Sonnenlande mit meinem Herzen ringt,

das ist die Sehnsucht nach dem Rhein, trautem Heimatstrom,

in dessen raunenden wellen sich spiegelt der Dom.

(10) 6. Strophe aus "Zum Abschied".

(11) 12. Strophe aus "Zum Abschied".

(12) 7. Strophe aus "Die Fahrt nach Curitiba", bezieht sich auf Vila velha.

(13) 1. Stropine aus "Zusammenbrucin 1945". 
LIRICA NO INTERIOR - UMA CONTRIBUIÇÃO $\bar{A}$ LITERATURA TEUTO-BRASILEIRA

Nas aldeias, nos nücleos de colonização alemã, nuitas vezes, encontram-se homens que se ocupam com as belas letras. Geralmente são camponeses, colonos, cuja tarefa diária é penosa. Seu tempo de dedicação à poesia é por isso sempre escasso. Na vida campestre representam algo de um centro cultural e intelectual. Participam de festividades comemorativas oficiais e familiares como oradores e fazem o papel de poetas ocasionais, exprimem e comentam em versos acontecimentos e fatos de seu ambiente. Tais atividades extraprofissionais não podem ser suficiente e altamente valorizadas na vida rústica.

ERICH KARL QUAST, de 73 anos, agricultor, natural da Alemanha, radicado nos arredores de Castro desde 1921, conforme a vontade de seu pai, devia dedicar-se a estudos teológicos, mas o destino deu-lhe um outro rumo. Escreve poesias, principalmente humorísticas, que, $\bar{a}$ primeira vista, parecem ser simples e des pretensiosas, mas mostram sabedoria e experiência de vida e contêm um significado escondido, i.èe, uma satira carinhosa em relação ao próximo e a fatos na aldeia.

Quast publicou em 1969 um fascículo de poesias de conteúdo humorístico, filosófico, baladescoe aforístico. Escreveu uma comédia com o título de "O chapéu novo", depois uma peça do mundo maravilhoso, "Três anões querem casar", em versos, para 
crianças. Essas pegas foram representadas por värias vezes no palco de Terra Nova. As poesias aqui orerecidas são ainda inéditas. Nota-se que nenhuma delas versa sobre temas do Brasil e da Alemanha, ao conträrio de outros poetas teuto-brasileiros como Carl. Fried, Karl Niederhut e Clemente Raul Einsiedel. Pais de origem e terra adoptiva não o inspiraram?

ANTON SCHULTE, nascido em 1915 na cidade de colónia, emigrou em 1935 para o Brasil e estabeleceu-se em Terra Nova, perto de Castro, onde vive como agricultor, dedicando-se com carinho à prosperidade da pequena comunidade alena. Oferecendo sua colaboração à igreja, escola e à associação cultural, Schulte, de natureza profundamente religiosa, $\vec{e}$ um homem conservador. Os antigos valores como fé, fidelidade, responsabilidade, devoção e espirito de sacrifício, têm para ele ainda enorme validade.

Näo sö a terra nativa, mas tambēm o Brasil, sua segunda pätria, oferecem-lhe os temas para seus versos. 